\title{
Evaluación de Polvos Alimentarios obtenidos de Cáscaras de Mango (Mangifera indica) como fuente de Ingredientes Funcionales
}

\author{
Liliana Serna-Cock ${ }^{(1)}$, Cristian Torres-León ${ }^{(1)}$ y Alfredo Ayala-Aponte ${ }^{(2)}$ \\ (1) Facultad de Ingeniería y Administración, Universidad Nacional de Colombia, Sede Palmira. Carrera 32 \\ Chapinero, Vía Candelaria, Bloque 25, 3o piso, oficina 3170. Palmira, Valle del Cauca, Colombia. \\ (2) Escuela de Ingeniería de Alimentos, Universidad del Valle, Cali.- Colombia \\ (e-mail: Iserna@unal.edu.co; ctorresl@unal.edu.co; alfredo.ayala@correounivalle.edu.co)
}

Recibido Jul. 14, 2014; Aceptado Sep. 23, 2014; Versión final recibida Oct. 6, 2014

\begin{abstract}
Resumen
Se evaluó el efecto del tamaño de partícula $(250 \mu \mathrm{m}$ y $180 \mu \mathrm{m})$ sobre la humectabilidad, solubilidad, capacidad de retención de agua (CRA), capacidad de retención de aceite (CRAC) e hinchazón en polvos liofilizados obtenidos de cáscaras de mango de las variedades criollo, Keitt, y Tommy Atkins. Además, se evaluó el contenido de antocianinas, carotenoides, licopeno y ácido ascórbico. El tamaño y la variedad influyeron significativamente $(p<0.05)$ sobre las propiedades funcionales de los polvos; a mayor diámetro, menor fue el tiempo de humectabilidad y mayor CRA y CRAC; y a menor diámetro mayor fue la solubilidad. El polvo de la variedad criollo mostró la mayor humectabilidad, solubilidad, contenido de antocianinas y ácido ascórbico. Las variedades Tommy Atkins y Keitt presentaron valores más altos de CRA (7.79-8.18 $\left.\mathrm{g}^{\mathrm{g}} \mathrm{g}^{-1}\right)$ y CRAC $\left(4.15-4.71 \mathrm{~g} \cdot \mathrm{g}^{-1}\right)$. El estudio muestra que los polvos de cáscara de mango pueden utilizarse para el desarrollo de alimentos funcionales.
\end{abstract}

Palabras clave: compuestos antioxidantes, diámetro de partícula, humectabilidad, solubilidad, Mangifera indica

\section{Evaluation of food powders obtained from peels of mango (Mangifera indica) as sources of functional ingredients}

\begin{abstract}
The effects of particle size (250 microns and 180 microns) on wettability, solubility, water holding capacity (WHC), oil holding capacity $(\mathrm{OHC})$, and swelling in freeze drying powders, obtained from three varieties of mango peels (Creole, Keitt and Tommy Atkins), were analyzed. In addition, the content of carotenoids, antioxianinas, and ascorbic acid, were evaluated. The particle diameter and the variety of mango affected the functional properties of the powders $(p<0.05)$. The higher was the particle diameter, the lower was the wettability and higher was the WHC and $\mathrm{OHC}$. As the particle diameter decreased, the solubility increased. The powder of criollo variety showed higher wettability, solubility, and content of anthocyanins and ascorbic acid. The Tommy Atkins and Keitt varieties had higher CRA values (7.79 to $\left.8.18 \mathrm{gg}^{-1}\right)$ and higher CRAC $\left(4.15\right.$ to $\left.4.71 \mathrm{gg}^{-1}\right)$. The study shows that mango peel powders can be used for the development of functional foods.
\end{abstract}

Keywords: retention capacity, antioxidant compounds, particle diameter, wettability, solubility, Mangifera indica 


\section{INTRODUCCIÓN}

Los polvos se utilizan ampliamente en la industria alimentaria por su estabilidad físico-química y microbiológica, porque aportan cualidades organolépticas, y contribuyen a mejorar las propiedades reológicas de los alimentos; además generan soluciones tecnológicas ya que son fáciles de conservar, transportar, almacenar, procesar, dosificar y utilizar (Cuq et al., 2013). La forma de las partículas, el tamaño, la porosidad, la composición y la densidad, determinan importantes propiedades funcionales tales como capacidad de retención de agua (CRA), capacidad de retención de aceite (CRAC), humectabilidad, velocidad de sedimentación, dispersabilidad y solubilidad, cualidades que influyen directamente en la calidad y aceptación del producto por parte de los consumidores (Cuq et al., 2011). La liofilización es un método apropiado para la obtención de polvos alimentarios, ya que produce una estructura porosa que absorbe el agua fácilmente, mejorando sus propiedades de rehidratación y conservando la mayor parte de las propiedades iniciales, incluyendo la concentración de compuestos bioactivos (Ceballos et al., 2012; Nora et al., 2014; Pérez et al., 2011).

En Colombia se producen 221.015 Tm de mango al año (FAOSTAD, 2011), la variedad criollo es la más solicitada para la elaboración de pulpas, las variedades florida (Tommy Atkins, Kent y Keitt) también se destacan por su utilización en procesamiento (pulpa, mínimamente procesado e I.Q.F), consumo en fresco y exportación (Asofrucol, 2012). Además de la utilización de la pulpa, se utiliza la cáscara pero en menor porcentaje para la fabricación de concentrados. En general la cáscara de mango es un residuo de alta contaminación del medio ambiente, además de su contenido de nitrógeno y fósforo tiene un alto contenido de agua que la hace susceptible a modificaciones por microorganismos creando problemas como la formación de lixiviados y la emisión de gases (Ajila et al., 2008). Por lo tanto, es una necesidad valorarla para uso alimentario, ya que es fuente de componentes nutricionales y bioactivos, que varían de acuerdo a la variedad. Según Ajila et al.,(2007a), las cáscaras de mango de las variedades Badami y Raspuri de la india son fuente de compuestos bioactivos, como los polifenoles, carotenoides, vitaminas, enzimas y fibras dietéticas. Por su parte Kim et al., (2010), reportan que la cáscara del mango de la variedad Irwin de Corea contiene más polifenoles y flavonoides que la pulpa, presentando actividad antioxidante efectiva. También se ha reportado altos contenidos de fibra dietética en mangos de la variedad Badami y Raspuri de la india (Ajila et al., 2007b). El objetivo de este trabajo fue evaluar el efecto del tamaño de partícula (250 y $180 \mu \mathrm{m})$ de polvos de cáscaras de mango liofilizado de tres variedades (criollo, keitt, y Tommy Atkins) sobre propiedades funcionales como la humectabilidad, solubilidad, capacidad de retención de agua (CRA), capacidad de retención de aceite (CRAC) e hinchazón. Además se evaluó el efecto de la variedad de mango sobre el contenido de compuestos antioxidantes (carotenoides, antocianinas, licopeno y ácido ascórbico), contenido de extracto etéreo y actividad de agua.

\section{MATERIALES Y MÉTODOS}

Materia prima: Se utilizaron 57 frutos de mangos (Mangifera indica) de la variedad criollo (conocido como mango de azúcar), con un peso por unidad de $138.13 \pm 17.74 \mathrm{~g}, 14$ frutos de la variedad keitt con un peso por unidad de $564.19 \pm 10.72 \mathrm{~g}$ ) y 15 frutos de la variedad Tommy Atkins con un peso por unidad de 518.42 $\pm 10.95 \mathrm{~g}$. Los mangos fueron proporcionados por la empresa frutícola Barahonda, de la ciudad de Palmira, del Departamento del Valle del Cauca (Colombia). En las tres variedades se utilizaron mangos maduros en estado de madurez 3 (ICONTEC, 2003, 2002). Los frutos se lavaron con agua potable y se sumergieron en agua clorada (100 ppm de hipoclorito de sodio) durante $10 \mathrm{~min}$, y se secaron con toalla de papel (Djioua et al., 2009). La cáscara se retiró con un pelador de cocina en acero inoxidabe, previamente desinfectado con hipoclorito de sodio 200 ppm. Se pesaron, se recolectaron en bolsas de polietileno (PE) estériles y se congelaron $\mathrm{a}-78^{\circ} \mathrm{C}$ por $24 \mathrm{~h}$, empleando un ultra congelador (New Bronswick, USA). Posteriormente, las cáscaras se liofilizaron (Labconco Freezone 4.5, USA) a presión de vacío de $133 \times 10^{-3} \mathrm{mBar}$, hasta alcanzar contenidos de humedad cercanos a $5 \%$ (b.h) y actividad de agua (aw) cercanos a 0,3. El contenido de humedad se determinó con un analizador por infrarrojos (Mettler Toledo AR-204, SUIZA), y la aw se midió a 24ํㅡ mediante un Aqua Lab (Decagon Devices, 3TE, USA). Las cáscaras secas se pulverizaron con un molino de cuchillas a 20000 rpm (IKA Labortechnik M20, CHINA). El material molido se pasó a través de un tamiz de $250 \mu \mathrm{m}$ y otro de $180 \mu \mathrm{m}$ en un Rotap (Tyler) durante $5 \mathrm{~min}$. La aw se midió se midió a 25드 mediante un Aqua Lab (Decagon Devices, Inc. Model Series 3TE, USA). Los polvos provenientes de las tres variedades de mango, y de los dos tamaños de partícula, se almacenaron en bolsas plásticas resellables a $5{ }^{\circ} \mathrm{C}$ para posterior uso. Todas las determinaciones de las propiedades funcionales se realizaron por triplicado.

Prueba de humectabilidad: Se utilizó la prueba de humectación estática, sugerida por Freudig et al., (1999) y modificada por Ceballos et al. (2012), utilizando un dispositivo cúbico de $5 \mathrm{~cm}$ de laterales internas. 
Solubilidad: Se determinó de acuerdo a lo reportado por Cano et al., (2005), con algunas modificaciones que se describen a continuación: a $50 \mathrm{ml}$ de agua destilada contenida en un tubo falcón se le adicionó $0,5 \mathrm{~g}$ de polvo de cáscara de mango, se llevó a una plancha de agitación (Corning PC - 420, USA), a 1150 rpm durante $5 \mathrm{~min}$. Posteriormente, la solución se colocó en un tubo falcón y se centrifugó a 3000 rpm durante 5 min. Una alícuota de $12,5 \mathrm{ml}$ del sobrenadante se transfirió a una placa de petri previamente pesada, las placas se secaron inmediatamente en estufa a $105^{\circ} \mathrm{C}$ durante $5 \mathrm{~h}$. El porcentaje de solubilidad se calculó por diferencia de peso según la ecuación 1.

Solubilidad en agua $(\%)=100\left(\frac{\text { peso sólidos en el sobrenadante } \times 4}{\text { peso de la muestra }}\right)$

Hinchazón: Se realizó de acuerdo con el método descrito por Robertson et al., (2000). La hinchazón se calculó como ml por g de polvo de cáscara de mango, utilizando la ecuación 2.

Hinchazón $\left(\frac{\mathrm{ml}}{\mathrm{g}}\right)=\frac{\text { volumen ocupado por la muestra }}{\text { Peso de la muestra }}$

Capacidad de retención de agua (CRA) y Capacidad de retención de aceite (CRAC): En tubos de $50 \mathrm{ml}$ se adicionaron $250 \mathrm{mg}$ de cáscara de mango en polvo, se adicionó agua destilada o aceite de oliva (25 ml) dependiendo de la medición a realizar CRA o CRAC. La mezcla se agitó y se dejó a $28^{\circ} \mathrm{C}$ durante $1 \mathrm{~h}$, se centrifugó (Eppendorf centrifuge 5804R, Germany) a $1500 \mathrm{~g}$ durante $10 \mathrm{~min}$, y posteriormente el sedimentó se pesó. CRA y CRAC se determinaron como g de agua o de aceite retenido por g de cáscara de mango, de acuerdo con el método descrito por Hassan et al., (2011)( Ecuación 3).

CRA/CRAC $\left(\frac{g}{g}\right)=\frac{\text { peso sedimento-peso muestra seca }}{\text { peso muestra seca }}$

Contenido de Extracto Etéreo: Se realizó para tener un soporte, para el análisis y discusión de la prueba de humectabilidad. Se determinó con un equipo de extracción de soxhlet de acuerdo al método 920.39 descrito por la (AOAC, 1990).

Determinación de antocianinas: Se prepararon extractos etanólicos de cada variedad, a $1 \mathrm{~g}$ de cáscara liofilizada se le adicionó $20 \mathrm{ml}$ de etanol al 80\%. Posteriormente se centrifugó a 5.000 rpm (Eppendorf centrifuge 5804R, Germany) durante $30 \mathrm{~min}$. La determinación se realizó según lo planteado por Lee et al., (2005), basado en el método diferencial de $\mathrm{pH}$, para esto el $\mathrm{pH}$ se ajustó con soluciones buffer de $\mathrm{pH} 1$ y $\mathrm{pH}$ 4.5, finalmente se realizaron lecturas a 520 y $700 \mathrm{~nm}$. La concentración de antocianinas se calculó a partir de la ecuación 4 , y se expresó en $\mathrm{mg} \cdot 100 \mathrm{~g}^{-1}$.

Antocianina $=\frac{A \times P M \times F D \times 10^{3}}{\varepsilon \times 1}$

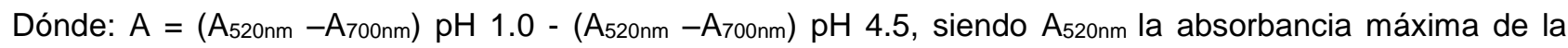
antocianina y $A_{700 n m}$ es la lectura de corrección debida a sustancias interferentes. PM (peso molecular) = $449.2 \mathrm{~g} / \mathrm{mol}$ cianidina-3- glucósido (antocianina mayoritaria), DF (factor de dilución) = 10, $1=$ longitud de la trayectoria en $\mathrm{cm}, \varepsilon=26900$ coeficiente de extensión molar, en $\mathrm{L} \times \mathrm{mol}^{-1} \mathrm{x} \mathrm{cm}^{-1}$ de la cianidina-3- glucósido, $10^{3}=$ Factor de conversión de g. $\mathrm{mg}^{-1}$.

Determinación del contenido de carotenoides y licopeno: A $1 \mathrm{~g}$ de polvo de cáscara liofilizada se le realizó la extracción de los compuestos antioxidantes, según la metodología planteada por Ordoñez et al., (2011). La determinación de carotenoides totales se realizó por espectrofotometría de acuerdo al método descrito por Higby, (1962). En una muestra de $4 \mathrm{ml}$ de fase orgánica (n-hexano) se leyó la absorbancia a $450 \mathrm{~nm}$, utilizando un espectrofotómetro (Thermo-Genesis $10 \mathrm{uv}$ ). El contenido de carotenoides, expresado en $\mathrm{mg} / 100 \mathrm{~g}$ de muestra, fue calculado mediante la ecuación 5.

Carotenoides Totales $\left(\frac{\mathrm{mg}}{100 \mathrm{~g}}\right)=\frac{\mathrm{A} 450 \times 100 \times \mathrm{V}}{250 \times \mathrm{L} \times \mathrm{W}}$

Dónde: 250 es el coeficiente de extinción $\left(\mathrm{L}_{\mathrm{g}} \mathrm{g}^{-1} \mathrm{~cm}^{-1}\right) ; \mathrm{L}=$ Longitud de la celda $(\mathrm{cm}) ; \mathrm{W}=\mathrm{cantidad}$ de muestra $(\mathrm{g})$ en el volumen final de la dilución y $\mathrm{V}=$ Volumen del solvente $(\mathrm{cm})$. 
La determinación de licopeno se realizó por espectrofotometría (Thermo-Genesis 10 uv). según ecuación 6, descrita previamente por (Barret \& Anthon, 2001).

$\frac{\mu \mathrm{l} \text { licopeno }}{\text { g peso fresco }}=\frac{\left(\mathrm{A}_{503} \times 537 \times \mathrm{V}\right)}{(\mathrm{W} \times 172)}$

Dónde: 537 es el peso molecular del licopeno $(\mathrm{g} / \mathrm{mol}), \mathrm{V}=$ volumen de la capa de hexano $(\mathrm{ml}), \mathrm{W}=$ cantidad de muestra $(\mathrm{g})$ y 172 es el coeficiente de extinción del licopeno en el hexano $\left(\mathrm{m}^{\mathrm{M}} \mathrm{M}^{-1}\right)$.

Determinación del contenido de Ácido ascórbico: Se diluyó 1 gramo de polvo liofilizado en $15 \mathrm{ml}$ de agua destilada, la determinación se realizó por reflectometria utilizando tiras de ensayo marca reflectoquant, en un reflectómetro (RQflex plus 10, Germany). El contenido de ácido ascórbico se expresó en $\mathrm{mg} / 100 \mathrm{~g}$ de muestra utilizando la ecuación 7.

Ácido ascórbico $\left(\frac{\mathrm{mg}}{100 \mathrm{~g}}\right)=\frac{\mathrm{L}^{*} \mathrm{~V}}{\mathrm{Pm}(\mathrm{ms})}$

Dónde: $\mathrm{L}=$ lectura $\left(\mathrm{mg} . \mathrm{l}^{-1}\right), \mathrm{V}=$ Volumen final $(\mathrm{ml}), \mathrm{Pm}=$ peso de la muestra $\mathrm{g}$ (b.s.)

Análisis estadístico: Para evaluar el efecto del tamaño de partícula sobre las propiedades funcionales de polvos obtenidos de cáscara de mango, se utilizó un diseño factorial al azar de $3^{\star} 2$, con dos factores: factor variedad de mango con tres niveles (criollo, Keitt y Tommy Atkins) y factor tamaño de partícula del polvo de mango con dos niveles (diámetro de partícula del polvo de 180 y de $250 \mu \mathrm{m}$ ). Las variables de respuesta fueron humectabilidad, solubilidad, capacidad de retención de agua, capacidad de retención de aceite e hinchazón. Para evaluar el efecto de la variedad de mango sobre la composición de compuestos antioxidantes, se utilizó un diseño unifactorial, siendo la variedad de la fruta el factor con los mismos tres niveles descritos anteriormente. Las variables de respuesta fueron composición de compuestos antioxidantes (carotenoides, licopeno, antocianinas y ácido ascórbico). Todos los análisis se realizaron por triplicado y se analizaron mediante análisis de varianza (ANOVA) con un nivel de confiabilidad del 95\%, para ello se empleó el programa SAS (SAS, 2012).

\section{RESULTADOS Y DISCUSIÓN}

Las cáscaras de mango presentaron contenidos de humedad de $81,593 \pm 0,1524 \%$ para la variedad Keitt, $82,808 \pm 0,282 \%$ para la variedad Tommy Atkins y 77,546 $\pm 0,1303$ para la variedad criollo. Al final del secado se presentaron valores de humedad inferiores al $6 \%$ resultados que son recomendados para mantener la estabilidad en los alimentos (Barbosa et al., 2007).

\section{Humectación}

La humectabilidad se define como la capacidad que tienen las partículas de adsorber agua en su superficie

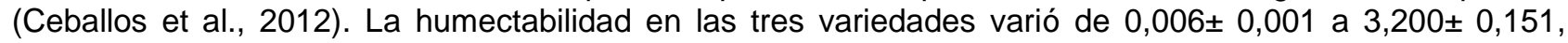
valores que están dentro de los rangos obtenidos por otros autores en polvos de jugos deshidratados (Papadakis et al., 2006). Se evidenció que hay relación inversa significativa $(p<0.05)$, entre el diámetro de partícula y la humectabilidad en el polvo de cáscara de mango en las tres variedades (Tabla 1), a mayor diámetro de partícula menor fue el tiempo de humectabilidad. Este comportamiento inverso puede ser atribuido a que al aumentar el tamaño de partícula se aumenta la cantidad de poros de la microestructura, y por consiguiente se aumenta la humectabilidad. De acuerdo a Dhanalakshmi et al., (2011), la humectación depende de factores micro estructurales como el tamaño de la partícula, la densidad y la porosidad. Según Forny et al. (2011), al aumentar el tamaño de la partícula se aumenta la cantidad de poros de la microestructura y al aumentar la porosidad se aumenta la humectabilidad. Estos resultados son de interés para la industria, ya que los polvos difíciles de humectar son inconvenientes para el consumidor y para el diseño de productos. Con respecto al factor variedad se observaron diferencias significativas $(p<0.05)$, la variedad Criollo presentó la mayor humectabilidad en ambos tamaños de partículas, debido posiblemente a que presentó menor contenido de extracto etéreo que las otras dos variedades (Tabla 1). La grasa disminuye la humectabilidad, debido a que favorece la hidrofobicidad superficial (Puri et al., 2010).

\section{Solubilidad}

La solubilidad se define como la velocidad y nivel en que los componentes de las partículas de polvo se disuelven en agua (Ortega, 2008); el porcentaje de solubilidad hace referencia a la cantidad de polvo que se deposita en el sobrenadante. Los valores de solubilidad de las cáscaras de mango se observan en la tabla 
1. Se observa que el comportamiento de la solubilidad es similar al de humectabilidad, mostrando significativamente $(p<0.05)$ que a menor tamaño de partícula, mayor es el porcentaje de solubilidad. Este resultado puede estar asociado al hecho de que a menor tamaño de partícula mayor es la velocidad de disolución. El factor variedad presentó también un efecto significativo $(p<0.05)$ sobre la solubilidad de los

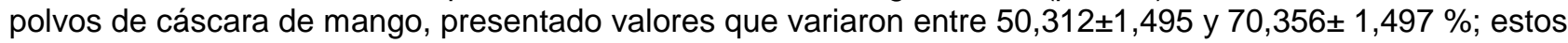
valores se consideran relativamente altos al compararse con cáscara de mango liofilizada de la variedad Tommy Atkins (53 a 64 \%) (Sogi et al., 2013). La variedad criollo presentó la mayor solubilidad en ambos tamaños de partícula, debido posiblemente a diferencias en su composición, es probable que contenga menores contenidos de fibra insoluble o mayor contenido fibra soluble respecto a los polvos de las otras variedades. Otras explicaciones pueden estar asociadas con la microestructras, dependiendo si la superficie de la partícula es amorfa o cristalina (Cano, et al., 2005); si predomina el estado amorfo mayor es la solubilidad de la partícula en agua (Gombas et al., 2003). Industrialmente la solubilidad es un criterio confiable para evaluar el comportamiento la calidad de un polvo en una solución acuosa (O'Shea et al., 2012).

\section{Hinchazón}

La hinchazón es una propiedad que afecta la humectabilidad de los polvos alimentarios (Schubert, 1993), se identifica como un factor que inhibe el hundimiento de los polvos (Freudig et al., 1999). En el presente estudio no se presentó hinchazón de los polvos de cáscara de mango en ninguna variedad y tamaño de partícula, a pesar que la literatura reporta valores de hinchazón en cáscaras de mango de la variedad Tommy $(5,57 \mathrm{ml} / \mathrm{g})$ (Vergara et al., 2007). Este resultado significa que la humectabilidad presentó valores óptimos. En los polvos alimentarios se requiere que las partículas se asienten lo suficientemente lento para que no formen sedimentos durante cierto periodo de tiempo. (O'Shea et al., 2012).

Tabla 1: Valores de humectabilidad, solubilidad Extracto etéreo y actividad de agua de polvos de cáscaras de mango de las variedades Criollo, Keitt y Tommy Atkins secadas por liofilización y con diámetros de partícula de $180 \mu \mathrm{m}$ y $250 \mu \mathrm{m}$. * Valores tomados después de la molienda. Todos los datos son la media \pm DS de tres repeticiones.

\begin{tabular}{|c|c|c|c|c|c|}
\hline Variedad & Tamaño $(\mu \mathrm{m})$ & Humectabilidad $(\mathrm{min})$ & Solubilidad $(\%)$ & Extracto Etéreo $(\%)$ & $a_{w}{ }^{*}$ \\
\hline \multirow{2}{*}{ Criollo } & 180 & $3.200 \pm 0.151$ & $70.356 \pm 1.497$ & $1.444 \pm 0.341$ & $0.410 \pm 0.008$ \\
\cline { 2 - 6 } & 250 & $0.017 \pm 0.005$ & $58.674 \pm 0.817$ & & $0.314 \pm 0.013$ \\
\hline \multirow{2}{*}{ Keitt } & 180 & $2.193 \pm 0.035$ & $68.717 \pm 0.652$ & $1.873 \pm 0.161$ & $0.337 \pm 0.002$ \\
\cline { 2 - 6 } & 250 & $0.004 \pm 0.001$ & $56.249 \pm 1.005$ & & $0.346 \pm 0.008$ \\
\hline \multirow{2}{*}{ Tommy Atkins } & 180 & $2.367 \pm 0.045$ & $61.009 \pm 3.063$ & $1.786 \pm 0.165$ & $0.298 \pm 0.030$ \\
\cline { 2 - 6 } & 250 & $0.006 \pm 0.001$ & $50.312 \pm 1.495$ & & $0.344 \pm 0.010$ \\
\hline
\end{tabular}

Los polvos después de la molienda presentaron valores de aw, ligeramente superiores a los medidos después del secado por liofilización, debido a la alta higroscopicidad de los productos; sin embargo todos los tratamientos presentaron valores de aw inferiores a 0.410 , lo cual indica que es estable en el almacenamiento contra ataque de microorganismos. Resultados similares se encontraron en pitahaya (Ayala et al., 2010).

\section{Capacidad de retención de agua (CRA) y Capacidad de retención de aceite (CRAC)}

En la Figura 1, se presentan los valores de CRA y de CRAC de las cáscaras de mango en las distintas variedades. En general, se observa que el diámetro de partícula de $250 \mu \mathrm{m}$ presenta mayores valores de CRA y de CRAC que el de $180 \mu \mathrm{m}$ en las tres variedades de mango, siendo la variedad Keitt la que presentó los mayores valores. De acuerdo al ANOVA, solo se presentaron diferencias significativas $(P<$ $0,05)$ en el tamaño de partícula para la CRAC. Estos resultados de CRA en cáscara de mango son superiores a los reportados por Sogi et al., (2013) con 4.68 en cáscaras de mango Tommy Atkins secadas por liofilización; mientras que los resultados de CRAC son relativamente similares a los reportados en cáscara de mangos de la variedad Hayden (Larrauri et al., 1996) y en cáscara de Manguifera pajang que es una fruta asiática pariente del mango (Hassan et al., 2011). 

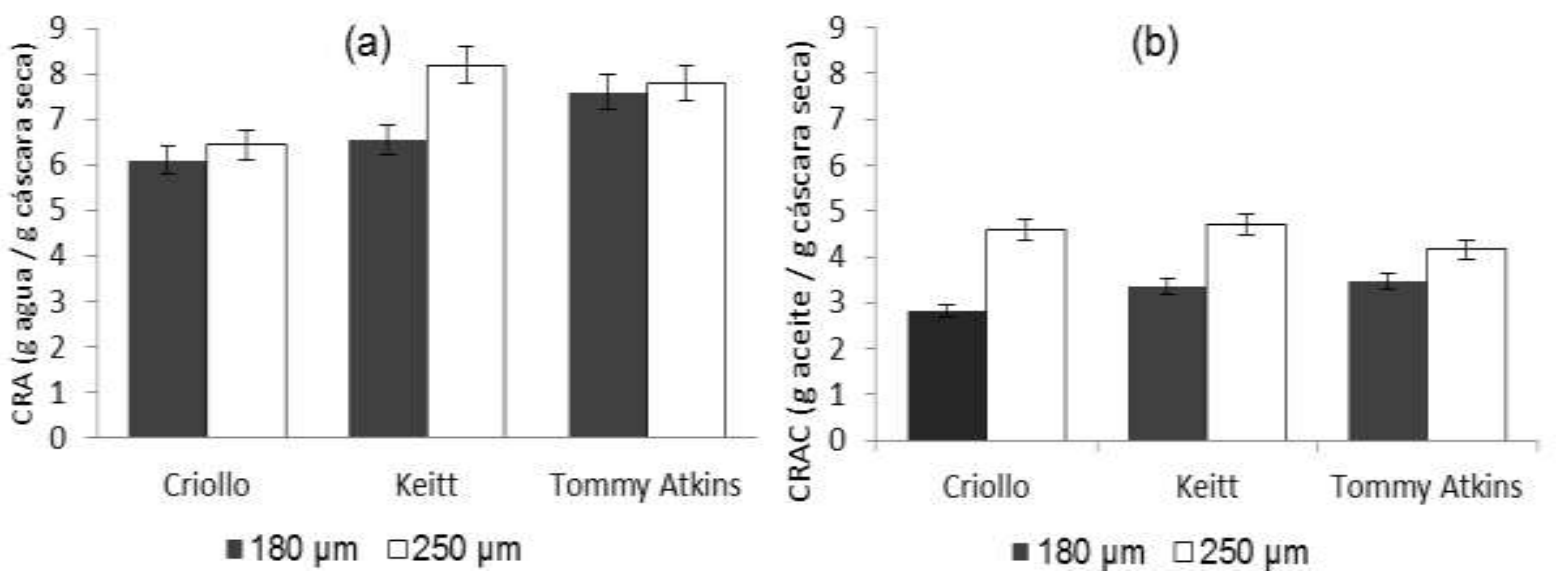

Fig. 1. Capacidad de retención de agua (CRA) (a) y capacidad de retención de aceite (CRAC) (b), en polvos de cáscaras de mango de las variedades Criollo, Keitt y Tommy Atkins secadas por liofilización y con diámetros de partícula de $180 \mu \mathrm{m}$ y $250 \mu \mathrm{m}$.

De acuerdo a estos resultados los polvos obtenidos de las cáscaras de mango tienen potencialidades en la industria alimentaria, por su capacidad de retención de aceite que es inclusive superior a la de los cereales comerciales (Falade et al., 2014), también pueden ser utilizados como aditivos en emulsiones, productos cárnicos y de panadería. Según Vergara et al., (2007), los valores de CRA y CRAC se incrementan al aumentar la temperatura, este comportamiento es de interés en la utilización de productos que requieran propiedades emulsionantes propias de las fibras vegetales y que estén asociados a incrementos de temperatura para su transformación. Por otro lado, polvos alimentarios con estas características de retención de aceites podrían utilizarse en la formulación de alimentos funcionales con efectos benéficos en la salud; de acuerdo a Elleuch et al. (2011), polvos alimentarios con estas cualidades pueden reducir el colesterol sérico.

\section{Extracto etéreo}

Puede observarse en la tabla 1, que los valores de extracto etéreo en las cascaras de las tres variedades variaron de $1.444 \pm 0,341 \%$ a $1.873 \pm 0,161 \%$, siendo la variedad keitt la que presentó el mayor valor.

\section{Compuestos antioxidantes}

En la tabla 2, se presentan los principales compuestos antioxidantes en los polvos de cáscaras de mango. El contenido de antocianinas en las cáscaras de mango de las tres variedades variaron significativamente $(\mathrm{p}<0.05)$ de 16,06 a $33,27 \mathrm{mg} .100 \mathrm{~g}-1$, siendo la variedad keitt la que presentó el menor contenido de antocianinas. Este resultado puede ser atribuido a la variación del color presentado en las cáscaras de los frutos, como vetas de color verde en las cáscaras de las variedades Tommy y Keitt. Estos valores de antocianinas en las tres variedades del fruto son superiores a los reportados por Sogi et al., (2013) en cáscaras de mango de la variedad Keitt con 3,3 (mg.100-1). A pesar que las determinaciones de antocianinas en cáscaras de mango son recientes, estos se pueden cuantificar para caracterizar su potencial antioxidante (Berardini et al., 2005). De acuerdo a estos resultados, la cáscara de mango de las tres variedades puede considerarse una fuente potencial de antioxidantes al compararse con otros frutos; en la literatura se reportan valores de 2,1 a 26,8 mg de cianidina 3-glucósido equivalente/100 g en cáscaras

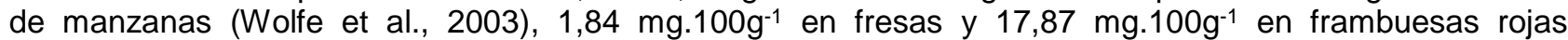
(mg.100g-1) y en uvas rojas (Samappito y Butkhup, 2010).

Tabla 2. Contenido de antocianinas, carotenoides, licopeno y de Acidó ascórbico en los polvos liofilizados de cáscara de mango. Los valores se expresan en peso seco, las antocianinas se expresaron como equivalentes de cianidina-3-glucosido. Todos los datos son la media \pm SD de tres repeticiones.

\begin{tabular}{|c|c|c|c|c|}
\hline Variedad & $\begin{array}{c}\text { antocianinas } \\
\left(\mathrm{mg.100g}^{-1}\right)\end{array}$ & $\begin{array}{c}\text { Carotenoides } \\
\left(\mathrm{mg.100g}^{-1}\right)\end{array}$ & $\begin{array}{c}\text { licopeno } \\
\left(\mathrm{mg}_{100 \mathrm{~g}^{-1}}\right)\end{array}$ & $\begin{array}{c}\text { Acidó ascórbico } \\
\left({\left.\mathrm{mg} .100 \mathrm{~g}^{-1}\right)}^{-1}\right.\end{array}$ \\
\hline Tommy Atkins & $19.329 \pm 3.05$ & $17.397 \pm 0.75$ & $1.828 \pm 0.05$ & $332.967 \pm 28.73$ \\
\hline Keitt & $16.026 \pm 0.73$ & $19.200 \pm 1.36$ & $1.946 \pm 0.12$ & $338.095 \pm 21.48$ \\
\hline Criollo & $33.275 \pm 6.07$ & $17.623 \pm 0.77$ & $1.837 \pm 0.14$ & $512.637 \pm 59.97$ \\
\hline
\end{tabular}


Los valores de carotenoides variaron entre 17.39 y $19.20 \mathrm{mg} .100 \mathrm{~g}^{-1}$ y el contenido de licopeno entre 1.82 y $1.94 \mathrm{mg} .100 \mathrm{~g}^{-1}$. La variedad keitt presentó ligeramente los mayores contenidos de carotenoides y de licopeno. Estos resultados en carotenoides fueron superiores a lo reportado en cáscara de mango Tommy Atkins, secadas por liofilización (4.05 mg.100g-1) (Sogi et al., 2013), similares a los reportados en cáscara de mango variedad indu Badami (19.4 mg.100g-1) (Ajila et al., 2007a) y menores a los encontrados en la variedad Raspuri (43.6-333.7 mg.100g-1) (Ajila et al., 2007b). Los resultados de licopeno fueron bajos en las tres variedades, resultado esperado ya que estos son abundantes en vegetales de color rojo como el Tomate Cherry, Sandia (Barba et al., 2006) y en tomate húngaro (Lugasi et al., 2003). A pesar de lo anterior, se cuantificó el licopeno debido a que las cáscaras de mango presentaron algunas trazas de coloración roja.

Con respecto al ácido ascórbico (Tabla 2), se observó que variaron significativamente $(p<0.05)$ de $332,967 \pm$ 28.73 a $512.637 \pm 59.97 \mathrm{mg} .100 \mathrm{~g}^{-1}$ en las tres variedades de mango, notándose que la variedad Criollo presentó el mayor contenido. Estos valores se consideraron relativamente altos al compararse con otros frutos, como la pulpa de manzana pera que cuenta con $257 \pm 11.7 \mathrm{mg} .100 \mathrm{~g}-1$ (p.f.) (Contreras et al., 2011), cáscara de naranja con $16.25 \mathrm{mg}$ de ácido ascórbico/100 g y cáscara de mandarina con 12.32 mg de acido ascórbico/100 g) (Rincón et al., 2005). Al compararse con cáscaras de mangos de distintas variedades se evidencia que la variedad criollo presentó mayor contenido de ácido ascórbico que lo reportado por Ajila et al., (2007b) en las variedades indues Raspuri (392 mg.g-1) y Badami (349 mg.g-1). Esta alta retención de ácido ascórbico en las cáscaras de las tres variedades pueden ser debidas al secado por liofilización, ya que este es considerado uno de los mejores métodos de conservación que ayuda a reducir la degradación térmica de la vitaminas (ácido ascórbico) en los vegetales (Ceballos et al., 2012). De acuerdo a los resultados de este estudio se evidenció que el polvo de cáscara de mango en las tres variedades evaluadas es una fuente potencial de compuestos antioxidantes. Las diferencias con los resultados de otras variedades de mango pueden estar asociados a distintas condiciones de maduración, variedad y país de origen (Ribeiro et al., 2008).

\section{CONCLUSIONES}

Se demostró que el diámetro de partícula y la variedad influyeron significativamente sobre las propiedades funcionales de los polvos alimentarios obtenidos de cáscara de mango. Cuanto mayor fue el diámetro de la partícula se evidenció menor tiempo en la humectabilidad y mayor CRA y CRAC, mientras que a menor diámetro de partícula, mayor fue la solubilidad, parámetro que se vio favorecido por el bajo contenido de extracto etéreo. El polvo de la cáscara de la variedad criollo mostró mayor humectabilidad, solubilidad, contenido de antocianinas y de ácido ascórbico, lo que indica que podría utilizarse para el diseño de productos funcionales; mientras que el polvo obtenido de las variedades Tommy Atkins y keitt presentaron mayor CRA (7.79 g.g-1 - 8.18 g.g-1) y CRAC (4.15 g.g-1- $4.71 \mathrm{~g} . \mathrm{g}-1$ ), lo cual podría tener aplicaciones industriales como agentes emulsionantes. De acuerdo a estos resultados, la obtención de polvo de cáscara de mango de las variedades Criollo, Tommy Atkins y keitt, presentan alto potencial de aplicación en la industria alimentaria.

\section{REFERENCIAS}

Ajila, C., Naidu, K., Bhat, S., Rao, U, Bioactive compounds and antioxidant potential of mango peel extract. Food Chemistry: 105 (3), 982-988. (2007a).

Ajila, C., Bhat, S., Rao, P, Valuable components of raw and ripe peels from two Indian mango varieties. Food Chemistry: 102 (4), 1006-1011. (2007b).

Ajila, C., Leelavathi, K., Rao, P, Improvement of dietary fiber content and antioxidant properties in soft dough biscuits with the incorporation of mango peel powder. Journal of Cereal Science: 48 319- 326 (2008).

AOAC. Official methods of analysis of the association of official analytical chemists. 15 Ed. Method 920.39. Arlington, Virginia, USA. (1990).

Asofrucol. Acuerdo de competitividad cadena productiva del mango en colombia. Bogota, Colombia. (2012).

Ayala A, Serna C., Mosquera E, Liofilización de pitahaya amarilla (Selenicereus megalanthus). Revista de la Facultad de química Farmaseutica: 17, 121-127. (2010).

Barba, A., Hurtado, M., Mata, M., Ruiz, V., Tejada, M, Application of a UV-vis detection-HPLC method for a rapid determination of lycopene and $\beta$-carotene in vegetables. Food Chemhemistry. 95, 328-336. (2006).

Barbosa, G., Fontana, J.,Schmidt, S., Labuza, T, Water activity in foods, First edit. ed. Blackwell Publishing, State Avenue, Ames, lowa, USA. (2006). 
Barret, D., Anthon, G, Lycopene content of California-grown tomato varieties. Acta H. 542, 165-173. (2001).

Cano, M., Stringheta, P., Ramos, A., Cal, J, Effect of the carriers on the microstructure of mango powder obtained by spray drying and its functional characterization. Innovative Food Science \& Emerging Technologies: 6 (4), 420-428 (2005).

Ceballos, A., Giraldo, G., Orrego, C, Effect of freezing rate on quality parameters of freeze dried soursop fruit pulp. Journal of Food Engineering: 111 (2), 360-365 (2012).

Contreras, J., Calderón, L., Guerra, E., García, B, 2011. Antioxidant capacity, phenolic content and vitamin C in pulp, peel and seed from 24 exotic fruits from Colombia. Food Research International. 44, 2047-2053.

Cuq, B.. Gaiani, C.. Turchiuli, C.. Galet, L.. Scher, L, Jeantet, R. Mandato, S. Petit, J. Murrieta, I. Barkouti, A. Schuck, P. Rondet, E. Delalone, M. Dumoulin, G. Delaplace, G. Ruiz, T. Chapter Two - Advances in Food Powder Agglomeration Engineering. Advances in Food and Nutrition Research: 69, 41-103. (2013).

Cuq, B., Rondet, E., Abecassis, J, Food powders engineering, between knowhow and science: Constraints, stakes and opportunities. Powder Technology: 208 (2), 244-251. (2011).

Dhanalakshmi, K., Ghosal, S., Bhattacharya, S, Agglomeration of food powder and applications. Critical reviews in food science and nutrition: 51 (5), 432-41. (2011).

Djioua, T., Charles, F., Lopez, F., Filgueiras, H., Coudret, A., Murillo F, Ducamp, Marie. Sallanon, H, Improving the storage of minimally processed mangoes (Mangifera indica L.) by hot water treatments. Postharvest Biology and Technology: 52 (2), 221-226. (2009).

Elleuch, M., Bedigian, D., Roiseux, O., Besbes, S., Blecker, C., Attia, H, Dietary fibre and fibre-rich byproducts of food processing: Characterisation, technological functionality and commercial applications: $\mathrm{A}$ review. Food Chem: 124, 411-421. (2011).

Falade, K., Semon, M., Fadairo, O., Oladunjoye, A., Orou, K, Functional and physico-chemical properties of flours and starches of African rice cultivars. Food Hydrocolloids: 39, 41-50. (2014).

FAOSTAD, Food and Agriculture organization of the united nations (2011). FAO, Retrieved am from http://faostat3.fao.org/faostat-gateway/go/to/download/Q/QC/S. Acceso 20 de enero de 2014.

Forny, L., Marabi, A., Palzer, S, Wetting disintegration and dissolution of agglomerated water soluble powders. Powder Technol: 206, 72-78. (2011).

Freudig, B., Hogekamp, S., Schubert, H, Dispersion of powders in liquids in a stirred vessel. Chemical Engineering and Processing: Process Intensification: 38 (4-6),525-532. (1999).

Gombas, A., Antal, I., Szabo, P., Marton, S. Eros, I, Quantitative determination of crystallinity of alphalactose monohydrate by near infrared spectroscopy (NIRS). I. Journal Pharmaceut: 256, 25-32.(2003).

Hassan, F. Ismail, A. Hamid, A. Al, S, Characterisation of fibre-rich powder and antioxidant capacity of Mangifera pajang K. fruit peels. Food Chemistry:126 (1), 283-288. (2011).

Higby, W, A simplified method for determination of some aspects of the carotenoid distribuition in natural and carotene fortified orange juice. journal of food science: $27,42-49$. (1962).

ICONTEC. Norma Técnica Colombiana 5139. Frustas frescas. Mangos criollos. Especificaciones. (2002).

ICONTEC. Norma Técnica Colombiana 5210. Frustas frescas. Mango variedades mejoradas. Especificaciones (2003).

Kim, H., Moon, J., Kim, H., Lee, D., Cho, M., Choi, H., Kim, Y,Mosaddik, A. Cho, S, Antioxidant and antiproliferative activities of mango (Mangifera indica L.) flesh and peel. Food Chemistry:121 429-436. (2010).

Larrauri, J., Rupérez, P., Borroto, B., Saura, F, Mango Peels as a New Tropical Fibre: Preparation and Characterization. Lebensmittel -Wissenschaft und -Technologie : 29, 729-733. (1996). 
Lee, J., Durst, R., Wrolstad, R, Determination of Total Monomeric Anthocyanin Pigment Content of Fruit Juices, Beverages, Natural Colorants, and Wines by the $\mathrm{pH}$ Differential Method: Collaborative Study. Journal of AOAC International: 88 (5) 1269-1278. (2005).

Lugasi, A., Bíro, L., Hóvárie, J., Sági, K., Brandt, S., Barna, E, Lycopene content of foods and lycopene intake in two groups of the Hungarian population. Nutrition Research: 23, 1035-1044 (2003)

Nora, C., Müller, C., Bona, G., Rios, A., Hertz, P., Jablonski, A., Jong, E., Flôres, S, Effect of processing on the stability of bioactive compounds from red guava (Psidium cattleyanum Sabine) and guabiju (Myrcianthes pungens). Journal of Food Composition and Analysis. 34, 18-25 (2014)

O'Shea, N., Arendt, E., Gallagher, E, Dietary fibre and phytochemical characteristics of fruit and vegetable by-products and their recent applications as novel ingredients in food products. Innovative Food Science \& Emerging Technologies: $16,1-10$. (2012).

Ordoñez, L., Vázquez, L., Romero, A, Micronutrient contents in organic and conventional tomatoes ( Solanum lycopersicum L.). International journal of science and technology. 46, 1561-1568. (2011).

Ortega, E., Bulk Properties of Food Particulate Materials: An Appraisal of their Characterisation and Relevance in Processing. Food Bioprocess Technology. 2, 28-44. (2008).

Papadakis, S.E., Gardeli, C., Tzia, C., Spray Drying of raisin juice concentrate. Drying Technology: 24 (2),173-180. (2006).

Pérez, M., Regueiro, J., González, C., Rial, R. Simal, J, Changes in antioxidant flavonoids during freezedrying of red onions and subsequent storage. Food Control: 22 (7), 1108-1113. (2011).

Puri, V., Dantulari, A., Kumar., M, Karar, N., Bansal., K, Wettability and surface chemistry of crystalline and amorphous forms of a poorly water soluble drug. E.Journal of Pharmaceutical Sciences. 40 (2) 84-93. (2010)

Ribeiro, S., Barbosa, L., Queiroz, J., Knödler, M., Schieber, A, Phenolic compounds and antioxidant capacity of Brazilian mango (Mangifera indica L.) varieties. Food Chemistry. 110 (3), 620-626. (2008).

Rincón, A., Vásquez, A., Padilla, C, composicion quimica y compuestos bioactivos de las harinas de cascaras de naranja (citrus sinensis), mandarina (citrus reticulata) y toronja (citrus paradisi) cultivadas en Venezuela. Archivos Latinoamericanos de Nutrición 55, 1-8.(2005).

Robertson, J., Monredon, F., Dysseler, P., Guillon, F., Amado, R., Thibault, F, Hydration Properties of Dietary Fibre and Resistant Starch: a European Collaborative Study. Food Science and T.: 33 (2),72-79. (2000).

Samappito, S. Butkhup, L, Analysis of Anthocyanin, Flavonoids, and Phenolic Acid Contents of Ten Fruits and Antioxidant Activity. International Journal of Fruit Science: 10, 264-280. (2010).

SAS. Statical analysis system. User's procedures guide. Version 9.3, cary: SAS institute, inc 2012.

Schubert, H, Foodstuffs in powder Instantization. : 33 International Chemical Engineering, 28-45. (1993).

Sogi, D., Siddiq, M., Greiby, I., Dolan, K,Total phenolics, antioxidant activity, and functional properties of "Tommy Atkins" mango peel and kernel as affected by drying methods. Food chemistry.141 (3), $2649-55$. (2013).

Vergara, N., Granados, E., Agama, E., Tovar, J., Ruales, J., Bello, L, Fibre concentrate from mango fruit: Characterization, associated antioxidant capacity and application as a bakery product ingredient. Food Science and Technology: 40 (4), 722-729. (2007).

Wolfe, K., Wu, X. Liu, R, Antioxidant activity of apple peels. J. of Agri. Food Chemistry: 3, 609-614 (2003). 
